Pesq. Vet. Bras. 36(7):625-629, julho 2016 DOI: $10.1590 / \mathrm{S} 0100-736 \mathrm{X} 2016000700011$

\title{
Aspectos epidemiológicos da leishmaniose visceral canina na zona rural do semiárido paraibano e análise de técnicas de diagnóstico ${ }^{1}$
}

\author{
Raizza B.S. Silva², Rodrigo S. Mendes², Vanessa L. Santana², Heitor C. Souza ${ }^{2}$, \\ Catarina P.S. Ramos ${ }^{4}$, Almir P. Souza ${ }^{2}$, Paulo P. Andrade ${ }^{5}$ e Marcia A. Melo ${ }^{2 *}$
}

\begin{abstract}
Silva R.B.S., Mendes R.S., Santana V.L., Souza H.C., Ramos C.P.S., Souza A.P., Andrade P.P. \& Melo M.A. 2016. [Epidemiological aspects of canine visceral leishmaniasis in the semi-arid region of Paraiba and analysis of diagnostic techniques.] Aspectos epidemiológicos da leishmaniose visceral canina na zona rural do semiárido paraibano e análise de técnicas de diagnóstico. Pesquisa Veterinária Brasileira. 36(7):625-629. Programa de Pós-Graduação em Medicina Veterinária, Universidade Federal de Campina Grande, Campus de Patos, Av. Universitária s/n, Bairro Santa Cecília, Patos, PB 58708-110, Brazil. E-mail: marcia.melo@pq.cnpq.br

Visceral leishmaniasis is caused by Leishmania infantum in Brazil. It is a chronic disease and often a fatal zoonosis and considered as a serious worldwide public health problem and recognized by the World Health Organization as one of seventeen "Neglected Tropical Diseases". This study was conducted in 2012 in the county of Patos, in Paraíba State semiarid region. The main purposes were to estimate the prevalence and the risk factors of canine visceral leishmaniasis (CVL), and to evaluate the performance of serological tests. Serological prevalence was determined using three techniques (ELISA, IFA and DPP chromatographic immunoassay - Dual Path Platform). ELISA was the golden standard, since it is the confirmatory test for CVL as established by the Brazilian Ministry of Health. In total, 362 blood samples were collected and were considered positive if positive by two serological assays. Positive samples were tested by PCR and qPCR. An epidemiological questionnaire was applied to the dog owner to identify risk factors associated with CVL. The seroprevalence was $11.33 \%$ (41/362). Chromatographic immunoassay - DPP showed a sensitivity of 58\% (95\%, CI: 0.43 to 0.72 ) and specificity of $96 \%$ (95\%, CI: 0.93-0.98) , with a Kappa index of 0.5. The IFA had a sensitivity of $85 \%(95 \%$, CI: 0.72 to 0.94$)$, specificity of $99 \%$ (95\%, CI: 0.98 to 1) and Kappa value of 0.8 . The risk factor identified in the multivariate analysis was gender (OR=2.15 [1.09 to 4.21]), male dogs having 2.15 times more chance of being infected. The increased likelihood can possibly be explained by the use of male dogs for hunting and as guard dogs, two factors that increase dog contact to infected sandflies. Due to the prevalence rates, it should be recommended control measures against canine visceral leishmaniasis. The results demonstrated that the Chromatographic immunoassay (DPP-Dual Path Platform) is not the best technique for screening positive animals due to its low sensitivity, suggesting its replacement by ELISA.
\end{abstract}

INDEX TERMS: Visceral leishmaniasis, neglected disease, endemic, Kinetoplastidae, Leishmania sp., dogs, Northeast, Brazil.

\footnotetext{
${ }^{1}$ Recebido em 14 de setembro de 2015.

Aceito para publicação em 24 fevereiro de 2016.

2 Programa de Pós-Graduação em Medicina Veterinária (PPGMV), Universidade Federal de Campina Grande (UFCG), Campus de Patos, Av. Universitária s/n, Bairro Sta. Cecília, Patos, PB 58708-110, Brasil. *Autor para correspondência: marcia.melo@pq.cnpq.br
}

\footnotetext{
${ }^{3}$ Curso de Graduação em Medicina Veterinária, UFCG, Campus de Patos, PB.

${ }^{4}$ Laboratório de Processos e Produtos Biotecnológicos (LPPB), Instituto de Ciências Biológicas, UPE, Rua Ambrósio Marques 310, Santo Amaro, Recife, PE 50100-130.

${ }^{5}$ Departamento de Genética, UFPE, Av. Professor Moraes Rego s/n, Cidade Universitária, Recife, PE 50732-970.
} 
RESUMO.- No Brasil, a leishmaniose visceral é causada pela Leishmania infantum. É uma zoonose crônica e, frequentemente, fatal, sendo considerada um grave problema de saúde pública no mundo e reconhecida pela Organização Mundial de Saúde como uma das dezessete "Doenças Tropicais Negligenciadas". 0 presente estudo foi realizado em 2012, na zona rural do município de Patos, semiárido paraibano, com o objetivo de estimar a prevalência, os fatores de risco da leishmaniose visceral canina (LVC) na região e avaliar os testes de diagnóstico sorológico. Foram coletadas 362 amostras de sangue e a prevalência da infecção foi determinada através de três técnicas sorológicas (ELISA, RIFI e Teste Imunocromatográfico DPP - Dual Path Plataform), sendo consideradas positivas as amostras que apresentaram pelo menos dois testes reagentes. As amostras positivas na sorologia foram testadas na PCR e qPCR. Aplicou-se um questionário epidemiológico aos proprietários para identificação dos possíveis fatores de risco. A taxa de prevalência foi de 11,33\% (41/362). Considerou-se o ELISA como padrão ouro, já que é o teste confirmatório para a LVC pelo Ministério da Saúde. O DPP (Dual Path Plataform) apresentou sensibilidade de $58 \%$ ( $95 \%$ CI: 0,43-0,72) e especificidade de $96 \%$ (95\% CI: 0,93-0,98), com índice Kappa de 0,57. A RIFI apresentou sensibilidade de 85\% (95\% CI: 0,72-0,94), especificidade de 99\% (95\% CI: 0,98-1) e valor Kappa de 0,88 . 0 fator de risco identificado na análise multivariada por regressão logística múltipla foi o sexo $(O R=2,15$ [1,09$4,21]$ ) e os cães machos apresentaram 2,15 vezes mais chances de serem infectados, que pode ser justificado por serem mais utilizados para a caça e como cão de guarda, dois fatores que aumentam a exposição do animal ao flebótomo. Em função da taxa de prevalência encontrada, indica-se a aplicação de medidas de controle no município. Os resultados da comparação dos ensaios sorológicos demonstraram que o DPP (Dual Path Plataform) não é a melhor técnica para triagem dos animais positivos em função da baixa sensibilidade do método, sugerindo que o mesmo seja substituído pelo ELISA.

TERMOS DE INDEXAÇ̃̃O: Leishmaniose visceral canina, doença negligenciada, endemia, Kinetoplastidae, Leishmania sp., Nordeste, Brasil.

\section{INTRODUÇÃO}

A leishmaniose visceral é uma zoonose crônica e, frequentemente, fatal, sendo considerada um grave problema de saúde pública no mundo e reconhecida pela Organização Mundial de Saúde como uma das dezessete doenças tropicais negligenciadas. No Brasil, é causada por Leishmania infantum, o principal vetor é o flebotomíneo Lutzomyia longipalpis e o cão é o principal reservatório doméstico (WHO 2014).

O Nordeste Brasileiro é a região com a prevalência mais alta de leishmaniose visceral humana (LVH) e canina (LVC) no país (Bavia et al. 2005). Vários fatores estão associados à prevalência, porém, são bastante controversos (Belo et al. 2013). Na Paraíba, pouco se sabe sobre a prevalência real da LVC nos municípios e quais os fatores de risco associados à infecção.

Apesar da grande variedade de métodos diagnósticos disponíveis, no Brasil, os testes sorológicos autorizados e re- comendados pelo Ministério da Saúde (MS) possuem sensibilidade e especificidade bastante variáveis. O MS substituiu o protocolo de diagnóstico da LVC, sendo utilizado atualmente o Teste Imunocromatográfico DPP (Dual Path Plataform, Biomanguinhos) como teste de triagem e o ELISA ${ }^{\circledR}$ - (Kit EIE Leishmaniose Visceral Canina - Biomanguinhos) como teste confirmatório, no lugar da reação de imunofluorescência indireta - Biomanguinhos (RIFI ${ }^{\circledR}$ ) (Brasil 2011). Falhas nesses resultados podem causar a eliminação animais não infectados, e, por outro lado, não detectar casos positivos, favorecendo a disseminação da doença.

Desta forma, o objetivo desse trabalho foi avaliar os fatores de risco da leishmaniose visceral canina na zona rural do município de Patos, PB, e verificar a sensibilidade e a especificidade dos métodos de diagnóstico disponíveis.

\section{MATERIAL E MÉTODOS}

O trabalho foi aprovado pelo Comitê de Ética do Centro de Saúde e Tecnologia Rural da Universidade Federal de Campina Grande, sob o protocolo no $53 / 2012$.

0 estudo foi realizado na zona rural do município de Patos, terceira maior cidade do estado da Paraíba, localizada no semiárido paraibano. Para estimativa da prevalência da leishmaniose visceral canina, a área estudada foi estratificada e a amostra total foi constituída de três estratos amostrais (norte, sul e oeste), com intuito de obter uma amostragem uniforme da área rural. Sorteou-se de forma aleatória simples sem reposição, um número pré-estabelecido de casas por estrato amostral da zona rural. 0 número de casas sorteadas foi determinado pela fórmula para amostras simples aleatórias, proposta por Thrusfield (2007), considerando uma prevalência esperada de $50 \%$, nível de significância de 5\% e erro amostral de 5\%.

Nas casas sorteadas foram realizadas coletas de sangue de todos os cães domiciliados (sem pré-requisitos quanto à idade, sexo ou raça), que representaram as unidades elementares do estudo. A coleta das amostras ocorreu em 2012. 0 sangue foi obtido por venopunção cefálica, com o auxílio de seringa de $5 \mathrm{~mL}$ BD e agulha 25x8mm BD estéril, de uso individual. Um total de $5 \mathrm{~mL}$ de sangue foi depositado imediatamente em tubo contendo o anticoagulante citrato de sódio $4 \%$.

Após a coleta, o material foi encaminhado para o Laboratório de Biologia Molecular do Semiárido do Centro de Saúde e Tecnologia Rural (CSTR), da Universidade Federal de Campina Grande (UFCG), campus Patos, PB. Foi retirado um mililitro de sangue total por amostra, feitas alíquotas, identificadas e congeladas a $-20^{\circ} \mathrm{C}$ para posterior utilização no diagnóstico molecular. As amostras restantes foram centrifugadas a $2000 x g$ por 10 minutos para separação do plasma, que foi transferido para microtubos de 1,5 mL, identificados e estocados a $-20^{\circ} \mathrm{C}$ até a realização dos ensaios sorológicos.

Os testes sorológicos foram realizados no Laboratório Municipal de Patos, PB, Laboratório Central de Saúde Pública da Paraíba (LACEN/PB), no Laboratório de Referência em Taxonomia e Diagnóstico de Reservatórios Silvestres das Leishmanioses (Instituto Oswaldo Cruz/RJ) e no Laboratório de Biologia Molecular do Semiárido (BIOMOL/UFCG). As técnicas utilizadas para o diagnóstico de infecção por Leishmania infantum nos cães foram: (a) Teste Imunocromatográfico DPP (Dual Path Plataform) Leishmaniose Visceral Canina (Biomanguinhos/FIOCRUZ); (b) Reação de imunofluorescência indireta (RIFI) com antígeno de formas promastigotas de Leishmania major-like MHOM/BR/76/JOF, (kit Leishmaniose Visceral Canina, Biomanguinhos/FIOCRUZ); (c) ELISA - (Kit EIE Leishmaniose Visceral Canina, Biomanguinhos/FIOCRUZ). Os controles foram estabelecidos a partir de cães sabidamente infectados e não infectados. Para todos os testes foi seguido o pro- 
tocolo do fabricante. Foram consideradas positivas para LVC as amostras que foram reagentes em dois testes sorológicos (Brasil 2006) e, posteriormente, foram testadas na Reação em Cadeia da Polimerase convencional (PCR) e na PCR em tempo real (qPCR).

A extração de DNA das amostras de sangue foi realizada empregando-se o reagente $\mathrm{Brazol}^{\circledR}$, seguindo as recomendações do fabricante ${ }^{6}$. Como controle positivo da PCR, utilizaram-se amostras de DNA extraídas de cultivo de L. infantum, mantidas em LIT, e caracterizada pelo perfil de isoenzima no Laboratório de Vigilância em Leishmanioses/ioc/FIOCRUZ. Após a extração, o DNA foi eluído em $50 \mu \mathrm{l}$ de tampão TRIS-EDTA (10mM Tris-Cl, pH 7,5 e $1 \mathrm{mM}$ de EDTA, pH 8,0) e a concentração foi medida em espectrofotômetro (Eppendorf/BioPhotometer plus). A amostra foi armazenada a $-20^{\circ} \mathrm{C}$ até o momento do uso.

Para a amplificação da sequência do minicírculo do kDNA de L. infantum foram utilizados os iniciadores LINR4 (5'-GGGGTTGGTGTAAAATAGGG-3') e LIN19 (5'-CAGAACGCCCCTACCCG-3'), que amplificam um produto de 720 pares de base (Aransay et al. 2000). A reação de amplificação continha 2 UI de Taq DNA polimerase, $10 \mathrm{mM}$ Tris- $\mathrm{HCl}, \mathrm{pH}$ 9,0; $50 \mathrm{Mm} \mathrm{KCl} ; 50 \mathrm{mM} \mathrm{MgCl}_{2}$ e $10 \mu \mathrm{M}$ dNTP. Cada reação foi realizada com a adição de $5 \mu \mathrm{L}$ do DNA extraído de cada amostra teste e $0,5 \mu \mathrm{M}$ de cada iniciador para um volume final de $20 \mu \mathrm{L}$. A termociclagem foi realizada em termociclador (BIOCYCLER MODELO), seguindo as seguintes etapas: desnaturação inicial de 5 minutos a $96^{\circ} \mathrm{C}$ seguida de 33 ciclos de $30 \mathrm{~s}$ a $95^{\circ} \mathrm{C}$, $30 \mathrm{~s}$ a $58^{\circ} \mathrm{C}$, e $1 \mathrm{~min}$ a $72^{\circ} \mathrm{C}$, seguido por um passo de extensão final por $7 \mathrm{~min}$ a $72^{\circ} \mathrm{C}$. 0 fragmento de DNA amplificado foi submetido à eletroforese em gel de agarose $1,2 \%$, por $50 \mathrm{~min}$ a uma voltagem de $80 \mathrm{~V}$ e corrente de $400 \mathrm{~mA}$. Os produtos amplificados foram corados com brometo de etídio a $0,5 \mu \mathrm{g} / \mathrm{ml}$, visualizados em transiluminador e fotografados.

A qPCR foi feita usando o termociclador da Bioer Technology. Os primers específicos Linf.1-23F (5'-TCCCAAACTTTTCTGGTCCT-3') e Linf.1-154R (5'-TTACACCAACCCCCAGTTTC-3') foram sintetizados de acordo com Cavalcanti et al. (2009) que amplificam um fragmento de 132 pares de base $\left(\mathrm{Tm} 81^{\circ} \mathrm{C}\right)$. A reação de amplificação continha $2 \mu \mathrm{l}$ de DNA das amostras, $1 \mathrm{mM}$ de cada primer e $6 \mu \mathrm{l}$ de SYBR $^{\circledR}$ Green PCR Kit (Qiagen), em volume final de $13 \mu$ l. A reação foi realizada usando uma etapa de desnaturação inicial de $95^{\circ} \mathrm{C}$ por 10 minutos, seguida de 40 ciclos de amplificação de $95^{\circ} \mathrm{C}$ por $15 \mathrm{~s} \mathrm{e}$ $60^{\circ} \mathrm{C}$ por $1 \mathrm{~min}$. Controles positivos e negativos contendo ou não o DNA de isolados de L. infantum foram incluídos. 0 software usado para a análise dos resultados foi o LineGeneK (versão 4.2.00).

Os cálculos estatísticos foram realizados com o programa SPSS 20.0 for Windows, com nível de significância de 5\%. As frequências relativa e absoluta dos animais reagentes na sorologia foram definidas por análise estatística descritiva. A concordância dos resultados obtidos nas técnicas sorológicas foi avaliada através da sensibilidade, especificidade e do indicador Kappa (k), conforme Andrade \& Zicker (1997). Para identificar os fatores de risco associados à soropositividade, foi realizada a análise univariada através do teste qui-quadrado de Pearson, ou Exato de Fisher quando necessário. Cada variável independente foi cruzada com a variável dependente e as que apresentaram valor de $p \leq 0,20$ (Zar 1999) foram selecionadas para a análise multivariada, utilizando-se a regressão logística múltipla (Hosmer \& Lemesho 2000).

\section{RESULTADOS}

Foram coletadas 362 amostras de sangue de cães da zona rural do município de Patos, $\mathrm{PB}$, e a prevalência sorológica da LVC nessa região foi de 11,33\%, ou seja, 41 dos 362 cães foram positivos em pelo menos dois testes sorológicos. A po-

\footnotetext{
${ }^{6}$ LGC Biotecnologia.
}

pulação estudada era composta por 247 machos $(68,23 \%)$ e 115 fêmeas (31,69\%). Quanto à raça, 94,48\% (342 cães) eram "sem raça definida" (SRD), com 39 soropositivos para LVC. Em relação ao contato com outros animais, 74,86\% tinham contato com outros cães, a maioria vivia em ambiente de terra e cimento $(66,30 \%)$ e 78,73\% eram cães de caça.

A técnica sorológica mais sensível foi o ELISA, que detectou 47 cães positivos e quatro ficaram na zona cinza (indeterminados), sendo considerados negativos para esse estudo. A RIFI e o DPP identificaram 42 animais positivos em cada teste. Para a análise e a comparação das técnicas sorológicas, considerou-se o ELISA como padrão ouro, já que é o teste confirmatório para o Ministério da Saúde (Brasil 2011). O DPP apresentou sensibilidade de 57,45\% (95\%, IC: 0,42-0,72) e especificidade de 95,56\% (95\%, IC: 0,93-0,98), com valor Kappa de 0,56, apresentando uma concordância moderada entre as técnicas. A RIFI apresentou sensibilidade de 85,11\% (95\%, IC: 0,72-0,94), especificidade de 99,37\% (95\%, IC: 0,98-1) e valor Kappa de 0,88, mantendo uma concordância quase perfeita quando comparado com o ELISA. 0 valor preditivo positivo (VPP), que é a probabilidade de ter a doença se o resultado for positivo, foi de $66,67 \%$ para o DPP e $95,24 \%$ para a RIFI. 0 valor preditivo negativo (VPN), que é a probabilidade de não ter a doença quando o resultado for negativo, foi de $93,75 \%$ e $97,81 \%$ para DPP e RIFI, respectivamente (Quadros 1, 2 e 3).

A PCR convencional, realizada nas amostras de sangue dos cães positivos na sorologia, amplificou um único fragmento de aproximadamente 720 pares de base (pb), confirmando a presença do parasito no sangue de 18 das 41 amostras testadas $(43,9 \%)$. Não foram observadas amplificações inespecíficas em nenhuma das amostras de campo. 0 único fragmento amplificado consiste no DNA de cinetoplasto de Leishmania infantum. Na qPCR, o índice de positivos subiu para 73,17\%, 30 das 41 amostras testadas amplificaram um fragmento de $132 \mathrm{pb}$.

Quadro 1. Valores absolutos (N) e porcentagens (\%) de amostras positivas e negativas no ensaio imunoenzimático indireto (ELISA), padrão ouro, e no teste imunocromatográfico DPP (Dual Path Plataform) para o diagnóstico da leishmaniose visceral canina na zona rural do município de Patos, PB

\begin{tabular}{ccccc}
\hline & & \multicolumn{2}{c}{ DPP } & \multirow{2}{*}{ Total } \\
\cline { 3 - 4 } & & Reagente (\%) & Não Reagente (\%) & \\
\hline \multirow{2}{*}{ ELISA } & Positivo & $28(7,73 \%)$ & $20(5,53 \%)-\mathrm{FN}^{\mathrm{a}}$ & 48 \\
& Negativo & $14(3,87 \%)-\mathrm{FP}^{\mathrm{b}}$ & $300(82,87 \%)$ & 314 \\
& Total & 42 & 320 & 362
\end{tabular}

$\overline{{ }^{\mathrm{a}} \mathrm{FN}}=$ falso-negativo, ${ }^{\mathrm{b}} \mathrm{FP}=$ falso-positivo.

Quadro 2. Valores absolutos (N) e porcentagens (\%) de amostras positivas e negativas no ensaio imunoenzimático indireto (ELISA), padrão ouro, e na reação de imunofluorescência indireta (RIFI) para o diagnóstico da leishmaniose visceral canina na zona rural do município de Patos, PB

\begin{tabular}{ccccc}
\hline & & \multicolumn{2}{c}{ RIFI } & \multirow{2}{*}{ Total } \\
\cline { 3 - 4 } & & Reagente (\%) & Não Reagente (\%) & \\
\hline \multirow{2}{*}{ ELISA } & Positivo & $40(11,05 \%)$ & $7(1,93 \%)-\mathrm{FN}^{\mathrm{a}}$ & 48 \\
& Negativo & $2(0,56 \%)-\mathrm{FP}^{\mathrm{b}}$ & $313(86,46 \%)$ & 314 \\
& Total & 42 & 320 & 362
\end{tabular}

$\overline{{ }^{\mathrm{a}} \mathrm{FN}=\text { falso-negativo, }}{ }^{\mathrm{b}} \mathrm{FP}=$ falso-positivo. 


\begin{tabular}{|c|c|c|c|c|c|c|}
\hline $\begin{array}{l}\text { Teste } \\
\text { iagnóstico }\end{array}$ & $\begin{array}{l}\text { Sensibilidade } \\
\text { (IC 95\%) }\end{array}$ & $\begin{array}{l}\text { Especificidade } \\
\text { (IC 95\%) }\end{array}$ & $\begin{array}{c}\mathrm{VPP} \\
\text { (IC 95\%) }\end{array}$ & $\begin{array}{c}\text { VPN } \\
\text { (IC 95\%) }\end{array}$ & $\begin{array}{l}\text { Índice } \\
\text { Kappa }\end{array}$ & $\begin{array}{l}\text { Conclusão da } \\
\text { replicabilidade }\end{array}$ \\
\hline PP & $\begin{array}{c}58,33 \% \\
(0,43-0,72)\end{array}$ & $\begin{array}{c}95,54 \% \\
(0,93-0,98)\end{array}$ & $\begin{array}{c}66,67 \% \\
(0,5-0,8)\end{array}$ & $\begin{array}{c}93,75 \% \\
(0,91-0,96)\end{array}$ & 0,57 & Moderada \\
\hline RIFI & $\begin{array}{c}85,11 \% \\
(0,72-0,94)\end{array}$ & $\begin{array}{l}99,37 \% \\
(0,98-1)\end{array}$ & $\begin{array}{c}95,24 \% \\
(0,84-0,99)\end{array}$ & $\begin{array}{c}97,81 \% \\
(0,96-0,99)\end{array}$ & 0,88 & Quase Perfeita \\
\hline
\end{tabular}

$\mathrm{Na}$ análise univariada dos fatores de risco, observou-se associação significativa $(\mathrm{p} \leq 0,2)$ para as variáveis sexo, raça, contato com cães, pequenos ruminantes, bovinos, ratos, ambiente onde vive, aptidão de caça e proximidade com açudes. Essas variáveis foram submetidas à análise multivariada (regressão logística múltipla) e o fator de risco identificado foi o sexo (Odds Ratio $=2,15$ ), em que os cães machos apresentaram 2,15 vezes mais chances de apresentar a doença.

\section{DISCUSSÃO}

0 nordeste brasileiro é a região com as mais altas taxas de prevalência de leishmaniose visceral humana e canina, e tem aumentado a frequência (Bavia et al. 2005). No município de Patos, $\mathrm{PB}$, no estudo atual, a taxa de prevalência encontrada na zona rural foi de $11,33 \%$, maior do que a observada na zona urbana em 2010, que foi de 7,24\% (Melo, 2016. Comunicação pessoal). A prevalência da LVC pode variar bastante entre as diversas localidades no mesmo município, o que sugere que os diversos ecossistemas favorecem a manutenção dos vetores de forma diferente e, em geral, a ocorrência na zona rural é maior do que na zona urbana (França-Silva et al. 2003); fato comprovado em estudo realizado por Porto (2010), que demonstrou que a prevalência na zona urbana de Patos foi de $7,2 \%$, diferença significativa em relação à zona rural no atual estudo, que foi de $11,33 \%$. Entre cidades e estados do Brasil a variação é ainda maior, com valores entre $0,7 \%$ em Salvador, na Bahia (Barboza et al. 2009), a 51,6\% na Ilha de São Luiz, no Maranhão (Silva-Abreu et al. 2008).

A sensibilidade do DPP ${ }^{\circledR}$, que detecta anticorpos contra rK26 e rK39, foi de apenas $57.45 \%$. 0 resultado é preocupante, pois com a tendência de aumento de prevalência observada nos últimos anos, e sendo a técnica de triagem utilizada pelo Ministério da Saúde para identificação dos animais positivos desde 2011, 42\% (IC 95\%: 0,28-0,57) dos cães permanecem no campo com resultado falso negativo. Vinte cães, positivos simultaneamente no ELISA e na RIFI, foram negativos no DPP. Esses animais permanecem como fonte de infecção para os mosquitos, favorecendo a disseminação da doença entre os animais e humanos, uma vez que o DPP é o teste de triagem recomendado pelo Ministério da Saúde e só serão testadas no ELISA, teste confirmatório do MS, as amostras que reagirem no primeiro ensaio. Queiroz Jr (2011), avaliando o DPP, encontrou resultado semelhante ao do presente estudo, com sensibilidade de $56,1 \%$ e especificidade de $100 \%$ quando comparado à RIFI. A sensibilidade variou com o quadro clínico do animal; 88,9\% em cães sintomáticos, 52,4\% em animais oligossintomáticos e de $12 \%$ em assintomáticos. Grimaldi Jr et al. (2012) também obtiveram resultados parecidos, cães sintomáticos com 98\% de sensibilidade e assintomáticos com $47 \%$.

Por outro lado, Gonçalves (2010) obteve um melhor desempenho no DPP (sensibilidade $=68,42 \%$, especificidade $=87,5 \%$ ), que se mostrou mais sensível, mais específico e com maior confiabilidade do que o ELISA e a RIFI, tendo como padrão ouro o exame parasitológico direto. Este fato pode ser em função da comparação com o exame parasitológico, que geralmente possui sensibilidade mais baixa do que os métodos sorológicos devido às características intrínsecas da técnica.

A RIFI, apesar de utilizar antígeno bruto (promastigotas de L. major-like), apresentou baixo índice de falsos positivos, apresentando uma especificidade de $99,37 \%$ e sensibilidade de 85,11\%. Segundo Schwanke et al. (2014), além da sensibilidade, a especificidade da RIFI pode ser influenciada pela natureza do antígeno.

Alguns cães positivos na sorologia foram negativos na PCR convencional e na PCR em tempo real. Isso pode acontecer devido a alguns fatores, como: (a) baixa quantidade de parasito circulante no sangue no momento da coleta, já que a densidade de Leishmania sp. no sangue periférico é baixa e a parasitemia é provavelmente episódica (Fichoux et al. 1999); (b) a presença de inibidores da PCR presentes no sangue, que podem afetar a sensibilidade do ensaio (Reithinger et al. 2003); (c) fatores relacionados aos primers; ao protocolo de extração do DNA e ao tipo de amostra utilizada (Reithinger et al. 2000, Lachaud et al. 2001); (d) permanência de anticorpos circulantes no sangue periférico mesmo após a eliminação do parasito; (e) reações cruzadas com outros patógenos na sorologia (Cabral 2007).

A sensibilidade da PCR é maior em amostras de DNA extraídas de medula óssea, baço e linfonodos, contudo a coleta dessas amostras é muito invasiva (Cabral 2007). Para estudos epidemiológicos em campo, programas de vigilância e controle da leishmaniose, a PCR a partir do DNA de sangue total de cães pode ser utilizada para complementar o resultado da sorologia (Maia et al. 2009).

A variável sexo foi apontada como fator de risco para a LVC, cães machos apresentaram 2,15 vezes mais chances de ter a doença (IC 95\%: 1,09-4,21; Valor de P: 0,0267; Erro padrão: 1,2484). Em uma revisão sistemática sobre os fatores de risco associados à LVC realizada por Belo et al. (2013), foi visto que as variáveis associadas à doença são bastante controversas e que vinte e três publicações associam a LVC com sexo. Em estudos epidemiológicos realizados em Tocantins (Santos 2008), Pernambuco (Dantas-Torres et al. 2006), na Croácia (Zivicnjak et al. 2005) e na Espanha (Fisa et al. 1999), cães machos apresentam maior 
prevalência da doença. Zivicnjak et al. (2005) apontaram que diferenças na prevalência poderiam ser devido a riscos distintos na exposição e/ou aumento na mortalidade feminina devido a gravidez e amamentação, resultando em maior renovação da população de fêmeas. No presente estudo, vários cães machos eram utilizados para a caça e normalmente são os preferidos como cão de guarda, dois fatores que aumentam a exposição ao flebótomo.

\section{CONCLUSÕES}

A prevalência sorológica da LVC na zona rural de Patos, $\mathrm{PB}$, foi de $11,33 \%$. Sugere-se que medidas de controle sejam adotadas no município.

Os resultados da comparação dos ensaios sorológicos demonstram que o DPP não é a melhor técnica para triagem em função da baixa sensibilidade do método, sugerindo que o mesmo seja substituído pelo ELISA.

\section{REFERÊNCIAS}

Andrade A.L.S.S. \& Zicker F. 1997. Avaliação de testes diagnósticos, p. 9-30. In: Andrade A.L.S.S. \& Zicker F. (Eds), Métodos de investigação epidemiológica em doenças transmissíveis. Vol.1. FNS, OPAS, Brasília, DF.

Aransay A.M., Scoulica E. \& Tselentis Y. 2000. Detection and identification of Leishmania DNA within naturally infected sand flies by seminested PCR on minicircle kinetoplastic DNA. Appl. Environ. Microbiol. 66(5):1933-1938.

Bavia M.E., Carneiro D.D., Gurgel H.C., Madureira Filho C. \& Barbosa M.G. 2005. Remote sensing and geographic information systems and risk of American visceral leishmaniasis in Bahia, Brazil. Parasitol. 47:165-169.

Barboza D.C.P.M., Leal D.C., Souza B.M.P.S., Carneiro A.J.B., Gomes Neto C.M.B., Alcântara A.C.D., Julião F.S., Moura S.A.B., Peralva L.M.P., Ferreira F. \& Franke C.R. 2009. Inquérito epidemiológico da leishmaniose visceral canina em três distritos sanitários do Município de Salvador, Bahia, Brasil. Revta Bras. Saúde Prod. Anim. 10(2):434-447.

Belo V.S., Struchinera C.J., Wernecka G.L., Barbosa D.S., Oliveira R.B., Neto R.G.T. \& Silva E.S. 2013. A systematic review and meta-analysis of the factors associated with Leishmania infantum infection in dogs in Brazil. Vet. Parasitol. 195:1-13.

Brasil 2006. Manual de vigilância e controle da leishmaniose visceral. Departamento de Vigilância Epidemiológica, Secretaria de Vigilância em Saúde, Ministério da Saúde, Brasília. 120p.

Brasil 2011. Esclarecimentos sobre substituição do protocolo diagnóstico da leishmaniose visceral canina (LVC). Nota Técnica Conjunta no 1, Coordenação Geral de Doenças Transmissíveis/Coordenação Geral de Laboratórios de Saúde Pública, Departamento de Vigilância das Doenças Transmissíveis, Secretaria de Vigilância em Saúde, Ministério da Saúde, Brasília. 3p.

Cabral A.W.D. 2007. Estudo comparativo entre o diagnóstico por técnicas sorológicas e da PCR para a detecção de Leishmania spp. Dissertação de Mestrado, Instituto de Biociências de Botucatu, Universidade Estadual Paulista. 56p.

Cavalcanti M.P., Brito M.E.F., Souza W.V., Gomes Y.M. \& Abath F.G. 2009. The development of a real-time PCR assay for the quantification of Leishmania infantum DNA in canine blood. Vet. J. 182:356-358.

Dantas-Torres F., Brito M.E. \& Brandão-Filho S.P. 2006. Seroepidemiological survey on canine leishmaniasis among dogs from an urban area of Brazil. Vet. Parasitol. 140: 54-60.

Fichoux Y.L., Quaranta J.F., Aufeuvre J.P., Lelievre A., Marty P., Suffia I., Rousseau D. \& Kubar J. 1999. Occurrence of Leishmania infantum Parasitemia in Asymptomatic Blood Donors Living in an Area of Endemicity in Southern France. J. Clin. Microbiol. 37(6):1953-1957.

Fisa R., Gállego M., Castillejo S., Aisa M.J., Serra T., Riera C., Carrió J., Gállego J. \& Portús M. 1999. Epidemiology of canine leishmaniosis in Catalonia (Spain) The example of the Priorat focus. Vet. Parasitol. 83: 87-97.
França-Silva J.C., Costa R.T., Siqueira A.M., Machado-Coelho G.L.L., Costa C.A., Mayrink W., Vieira E.P., Costa J.S., Genaro O. \& Nascimento E. 2003. Epidemiolgy of canine visceral leishmaniasis in the endemic area of Montes Claros Municipality, Minas Gerais state, Brazil. Vet. Parasitol. 111:161-173.

Gonçalves B.S. 2010. Leishmaniose visceral canina na área urbana de Cuiabá MT: comparação de técnicas laboratoriais, tentativa de desenvolvimento de metodologia para o diagnóstico e caracterização da espécie de Leishmania circulante em amostra selecionada. Dissertação de Mestrado Acadêmico em Ciências Biológicas, Escola Nacional de Saúde Pública Sergio Arouca (ENSP), Fundação Oswaldo Cruz (FIOCRUZ), Rio de Janeiro, RJ. 125 p.

Grimaldi Jr G., Teva A., Ferreira A.L., Santos C.B., Pinto I.D., Azevedo C.T. \& Falqueto A. 2012. Evaluation of a novel chromatographic immunoassay based on Dual-Path Platform technology (DPP ${ }^{\circledR}$; CVL rapid test) for the serodiagnosis of canine visceral leishmaniasis. Trans. R. Soc. Trop. Med. Hyg. 106(1):54-59.

Hosmer D.W. \& Lemesho W.S. 2000. Applied Logistic Regression. 2nd ed. John Wiley and Sons, New York, 375p. Disponível em <http://books. google.com.br/books?id=Po0RLQ7USIMC\&printsec=frontcover\&$\mathrm{hl}=\mathrm{pt}-\mathrm{BR} \&$ source=gbs_ge_summary_r\&cad $=0 \# \mathrm{v}=$ onepage \&q\&f=false> Acesso em out. 2013.

Lachaud L., Chabbert E., Dubessay P., Reynes J., Lamothe J. \& Bastien P. 2001. Comparison of various sample preparation methods for PCR diagnosis of visceral leishmaniasis using peripheral blood. J. Clin. Microbiol. 39:613-617.

Maia C., Ramada J., Cristóvão J.M., Gonçalves L. \& Campino L. 2009. Diagnosis of canine leishmaniasis: Conventional and molecular techniques using different tissues. Vet. J. 179:142-144.

Melo M.A. 2016. Comunicação pessoal (Programa de Pós-Graduação em Medicina Veterinária, Universidade Federal de Campina Grande, Campus de Patos, Av. Universitária s/n, Bairro Sta. Cecília, Patos, PB 58708-110, Brasil.

Porto M.L. 2010. Soroprevalência e fatores de risco para Leishmaniose Visceral Canina em Patos, Paraíba, Brasil. Trabalho de conclusão de curso (Graduação em Medicina Veterinária), Universidade Federal de Campina Grande (UFCG), Patos, PB. 46 p

Queiroz Jr E.M. 2011. Validação do teste imunocromatográfico rápido Dual Path Platform para o diagnóstico da leishmaníase visceral canina. Dissertação de Mestrado em Ciências Veterinárias, Faculdade de Veterinária, Universidade Estadual do Ceará, Fortaleza. 77p.

Reithinger R., Lambson B.E., Barker D.C. \& Davies C.R. 2000. Use of PCR to detect Leishmania (Viannia) spp. in dog blood and bone marrow. J. Clin. Microbiol. 38:748-751.

Reithinger R., Espinoza J.C., Courtenay O. \& Davies C.R. 2003. Evaluation of PCR as a diagnostic mass-screening tool to detect Leishmania (Viannia) spp. in domestic dogs (Canis familiaris). J. Clin. Microbiol. 41:1486-1493.

Santos H.D. 2008. Fatores Associados à Soropositividade para Leishmaniose Visceral Canina no Município de Piraquê, Estado do Tocantins, Brasil. Tese de Doutorado em Ciências Veterinárias. Universidade Federal Rural do Rio de Janeiro, Rio de Janeiro, RJ, Brasil. 89 p.

Schwanke K., Silva A.M.M., Pacheco A., Bahia M., Silveira F.T., Scofield A. \& Góes-Cavalcante G. 2014. Diagnóstico molecular e frequência de anticorpos anti-Leishmania infantum chagasi em cães do município de Belém, Pará. Pesq. Vet. Bras. 34(3):255-260.

Silva-Abreu A.L., Lima T.B., Macedo A.A., Moraes-Júnior F.J., Dias E.L., Batista Z.S., Calabrese K.S., Moraes J.L.P., Rebêlo J.M.M. \& Guerra R.M.S.N.C. 2008. Soroprevalência, aspectos clínicos e bioquímicos da infecção por leishmania em cães naturalmente infectados e fauna de flebotomíneos em uma área endêmica na ilha de São Luís, Maranhão, Brasil. Revta. Bras. Parasitol. Vet. 17:197-203.

Thrusfield M. 2007. Veterinary Epidemiology. Wiley Blackwell, Oxford. 610p.

WHO 2014. Leishmaniasis. World Health Organization, Geneva. Disponível em <http://www.who.int/mediacentre/factsheets/fs375/en/> Acesso em out. 2014.

Zar J.H. 1999. Biostatistical Analysis. 4th ed. Prentice-Hall, New Jersey. 663p. Zivicnjak T., Martinković F., Marinculić A., Mrljak V., Kucer N., Matijatko V., Mihaljević Z., Barić-Rafaj R. 2005. A seroepidemiologic survey of canine visceral leishmaniosis among apparently healthy dogs in Croatia. Vet. Parasitol. 131: 35-43. 